\title{
Licht und Schatten digitaler Transparenz
}

Höhere Transparenz durch digitale Technologien gilt als Königsweg, die individuelle und organisationale Leistungsfähigkeit zu verbessern. Aus einer HR-Perspektive bietet sie so neue Chancen für die Motivation und Entwicklung von Mitarbeitern. Diese sollten jedoch nicht losgelöst von potenziellen Schattenseiten digitaler Transparenz betrachtet werden.

Till Remmers, Daniel Schaupp 
Forderungen nach mehr Transparenz in Organisationen sind allgegenwärtig. War „vollkommene Transparenz" in Organisationen lange nicht mehr als eine Utopie, scheint sie heute dank neuer digitaler Technologien in zunehmendem Maße realisierbar. Dabei gilt Transparenz, gestützt auf entsprechende Argumente der Management-Forschung, als Lösung für vielfältige innerbetriebliche Probleme (vergleiche beispielsweise Bennis/Goleman/ O’Toole 2008).

Digitale Transparenz, also Transparenz geschaffen durch digitale Technologien, macht Informationen in bisher ungekanntem Ausmaß verfügbar. Damit unterstützt sie sowohl den einzelnen Mitarbeiter als auch die Organisation in ihrer Gesamtheit dabei, den Überblick über die Geschäftstätigkeit zu behalten, eigene Leistung einzuordnen und Fähigkeiten weiterzuentwickeln. Insbesondere für moderne Ansätze der Mitarbeitermotivation und -entwicklung ist eine solche Transparenz essenziell.

Dementsprechend überrascht es nicht, dass Vorstände, sowohl in Deutschland als auch international, die Förderung von digitaler Transparenz ganz oben auf ihre Agenda setzen (vergleiche zum Beispiel Deloitte 2016; EIU 2018). Entsprechend bewerben auch Anbieter von Business-Intelligence (BI)-Systemen, Big Data Solutions und Advanced-Analytics-Anwendungen ihre Produkte.

In dieser sich selbst verstärkenden Euphorie bleibt hingegen häufig unklar, was „mehr Transparenz“ eigentlich bedeutet und welche Folgen ein Mehr an solcher Transparenz für die Organisation in ihrer Gesamtheit und den einzelnen Mitarbeiter tatsächlich hat. So stehen sich oftmals begeisterte Utopien und ähnlich drastische Dystopien - man denke an Dave Eggers prominent verfilmten Roman „The Circle“ (2015) - gegenüber, während eine unvoreingenommene Betrachtung alles andere als trivial erscheint.

Umso wichtiger ist es, den Begriff der Transparenz genau abzugrenzen sowie forschungsgestützt Einflussfaktoren auf und Konsequenzen von Transparenz zu identifizieren.

\section{Grundlagen von Transparenz}

Ein Grund für die Popularität von Transparenz ist die gängige Assoziation mit anderen positiv belegten und ähnlich vagen Begriffen wie Offenheit und Fairness, welche sich sowohl in der breiten Öffentlichkeit als auch in der Wissenschaft beobachten lässt. Dieser Umstand war im akademischen Diskurs der vergangenen Jahre Ausgangspunkt für eine genauere, disziplinenübergreifende Auseinandersetzung mit dem Begriff Transparenz mit zwei wesentlichen Erkenntnissen. Wenn von innerbetrieblicher Transparenz gesprochen wird, ist erstens häufig der Zugang zu relevanten, verständlichen und präzisen Informationen gemeint (vergleiche Schnackenberg/ Tomlinson 2014). Eine Forderung nach mehr Transparenz ist somit im Kern eine Forderung nach einer verbesserten Informationsausstattung. Transparenz ist zumeist positiv konnotiert, weil sie zweitens aus Sicht der Beobachtenden, also derjenigen, denen etwas transparent gemacht wird, beurteilt wird. Entsprechend spielen alternative Blickwinkel sowie die zugrunde

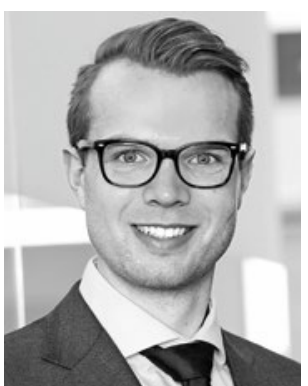

\section{Till Remmers}

ist Research Assistent und promoviert am Institut für Management \& Controlling an der WHU - Otto Beisheim School of Management in Vallendar.

E-Mail: till.remmers@whu.edu

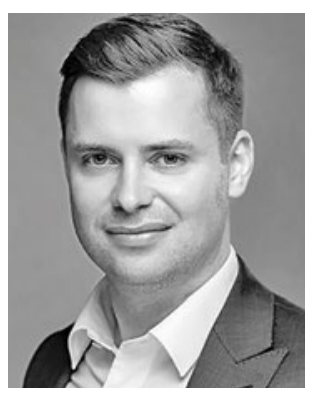

Ass. Prof. Dr. Daniel Schaupp ist Assistant Professor an der WHU Otto Beisheim School of Management in Vallendar.

E-Mail:daniel.schaupp@whu.edu 
Transparenz kann als Zugang zu relevanten, verständlichen und akkuraten Informationen definiert werden. liegenden Mechanismen in der Bewertung häufig nur eine untergeordnete Rolle (vergleiche Bernstein 2017).

\section{Vorteile von Transparenz}

Transparenz ermöglicht den Beobachtenden Lernen und Kontrolle. KPIDashboards in BI-Systemen machen ihnen basierend auf einer Vielzahl unternehmensweit gesammelter Daten Informationen in grafischer, einfach zu bedienender Form zugänglich. Sie helfen ihnen so beispielsweise durch individuell konfigurierbare Reports und Analysen dabei, die individuelle Leistung einzuordnen und Verbesserungspotenziale zu identifizieren. Sie gestatten zudem der Organisation, Unternehmensziele zu kommunizieren sowie deren anschließende Umsetzung zu kontrollieren. Durch breite Verfügbarkeit, abgestimmte Dashboards und Möglichkeiten zum einfachen Visualisieren eigener Analysen ermöglichen sie zusätzlich Lernen auf organisationaler Ebene. Potenziell kann diese Transparenz in zunehmendem Maße überall (in Form mobiler Geräte) und unmittelbar (in der Auswertung von Echtzeit-Daten) umgesetzt werden.

Die genannten Vorteile gelten unabhängig von der Art der Beobachtenden, seien es einzelne Mitarbeiter oder eine ganze Business Unit. Ein Blick auf die verhaltensorientierte Forschung zeigt zudem, dass Transparenz positive psychologische Auswirkungen für die Beobachtenden haben kann: Etwas transparent gemacht zu bekommen vermittelt ein Gefühl von Sicherheit und Kontrolle, Gemeinsamkeit, persönlicher Bedeutung und Einfluss. Diese wirken sich schlussendlich auch positiv auf die individuelle und organisationale Leistung aus.

\section{„Transparenz kann in zunehmendem Maße überall und unmittelbar umgesetzt werden."}

Das, was als Vorteil gilt, hat sich in den vergangenen Jahren signifikant verschoben. War Transparenz in Unternehmen früher dediziert vertikal und asymmetrisch ausgerichtet, um maximale Kontrolle „,von oben nach unten“ zu gewährleisten, wird diese heute zunehmend stärker auch horizontal und symmetrisch („von links nach rechts") eingesetzt (vergleiche Heald 2006). Damit können Potenziale von Transparenz auch in der Breite freigesetzt werden. Aus einer HR-Perspektive wird häufig nur der horizontale Ansatz überhaupt als ein "transparentes Arbeitsumfeld“ verstanden, da er weniger auf Kontrolle und mehr auf Befähigung der einzelnen Mitarbeiter ausgerichtet ist. In diesem Zusammenhang kann den einzelnen Mitarbeitern auch ermöglicht werden, aktiv ihren Transparenzrahmen, das heißt ihren Zugang zu Informationen, zu erweitern, neue Informationen zu entdecken und Daten abzufragen. Kommunizieren Unternehmen Transparenz dementsprechend, können sie diese nutzen, um eine gemeinsame Unternehmenskultur $\mathrm{zu}$ entwickeln. So interpretiert scheint es tatsächlich nicht zu viel Transparenz geben zu können. 


\section{Transparenz und individuelle Privatsphäre}

Die Erhebung und Speicherung von Informationen, die mit einer solchen Transparenz einhergehen, ist allerdings auch der erste Schritt in Richtung der Kehrseite digitaler Transparenz. Um individuelle und organisationale Transparenz zu ermöglichen, ist eine möglichst weitreichende Datenerfassung notwendig. Der „Data Lake“ als Sammelplatz vielfältigster, zweckungebundener Daten verkörpert diesen Bedarf sinnbildlich. Solch eine weitreichende Datenerfassung innerhalb einer Organisation hat jedoch immer auch einen Personenbezug, ob direkt wie bei Verkaufszahlen eines Vertriebsmitarbeiters oder indirekt in Form von Maschinenausfallzeiten oder Projekterfolgen beziehungsweise -misserfolgen. Wo ein Beobachter, da ist auch stets mindestens ein Beobachteter.

Für Beobachtete gestaltet sich die Einordnung der Transparenz deutlich ambivalenter. Zum einen ist ein gewisses Maß an Sichtbarkeit innerhalb einer Organisation notwendig und bietet die Gelegenheit, sich selbst zu präsentieren. So motiviert Transparenz regelmäßig zu stärkeren Anstrengungen. Zum anderen ist Transparenz jedoch immer auch besorgniserregend, insbesondere wenn sie zunehmend weiter ausgreift. Aus ökonomischer Perspektive geht gegebenenfalls ein wertvoller Informationsvorsprung verloren. Aus psychologischer Sicht kann allein die Möglichkeit, für andere transparent zu sein, beträchtliche psychologisch-motivationale Konsequenzen haben. Mit der zunehmenden Sammlung von Informationen in Unternehmen, die einen persönlichen Bezug haben, geht die Kontrolle über die eigene informatorische Privatsphäre verloren. Diese Privatsphäre ist für den Einzelnen jedoch psychologisch so wertvoll wie die Transparenz selbst. Sie vermittelt ein Gefühl der Sicherheit und Wertschätzung, was ebenfalls motivierend ist und sich positiv auf die individuelle Leistungsfähigkeit auswirkt (vergleiche Bhave/Teo/Dalal 2019).

Die empfundene Bedrohung der informatorischen Privatsphäre kann dazu führen, dass die Mitarbeiter versuchen, der Transparenz aus dem Weg zu gehen oder diese gezielt zu ihren Gunsten zu beeinflussen. Wo dies nicht möglich ist, reagieren Mitarbeiter häufig mit „Dienst nach Vorschrift“, selbst wenn ihnen bessere Alternativen bekannt sind. Dieses TransparenzManagement bindet Ressourcen, führt zu verfälschten Darstellungen, beiderseitigem Vertrauensverlust und Frustration. So zeigen die Ergebnisse einer Studie, dass Mitarbeiter erhebliche Aufmerksamkeit darauf verwenden, Beobachtung durch die Organisation festzustellen und auf jene entsprechend zu reagieren. Im Gegensatz dazu wurde nach Prozessverbesserungen weniger intensiv gesucht und gefundene Verbesserungen nicht untereinander geteilt, um nicht durch abweichende Tätigkeit aufzufallen (vergleiche Bernstein 2012).

In der Gesamtsicht gestaltet sich die Einordnung der Transparenz für die einzelnen Mitarbeiter besonders komplex und vielschichtig, da sie in einer Organisation immer sowohl Beobachter als auch Beobachtete sind. Positive und negative Konsequenzen prallen somit aufeinander. Es kommt zu einer Inkongruenz, die mögliche negative Leistungs- und Motivationswirkungen poten-

\section{Transparenz ermöglicht Lernen und Kontrolle für Einzelpersonen und Organisationen.}

\section{Zusammenfassung}

- Transparenz durch digitale Technologien ermöglicht es Organisationen und deren Mitarbeitern, den Überblick über die Geschäftstätigkeit zu behalten und die individuelle Leistungsfähigkeit zu verbessern.

- Zugleich verringert digitale Transparenz jedoch den Raum für Privatsphäre der Mitarbeiter und führt potenziell zu einer neuen Intransparenz im Hinblick auf die digitalen Technologien.

- Vor- und Nachteile müssen in der Ausgestaltung der Unternehmenskultur, der Leistungsbeurteilung und -entwicklung sowie technischen Umsetzung berücksichtigt werden, um tatsächliche Vorteile von Transparenz realisieren zu können. 
ziell noch verstärkt, da sie zusätzliche Aufmerksamkeit und Ressourcen der Mitarbeiter beansprucht. Für die Organisation als Ganzes bedeuten die individuellen Aktivitäten unterschiedliche Verhaltensmuster, die entweder organisationales Lernen, Motivation und Leistung ins Zentrum rücken oder solche, die Passivität, Demotivation und Leistungsminderung beinhalten.

\section{Ein tiefes Verständnis transpa- renzsteigernder digitaler Technologien verhindert} neue Intransparenz.

\section{Neue digitale Intransparenz}

Digitale Technologien versuchen, vollständige Transparenz zu ermöglichen, indem sie bisherige, menschliche Limitationen überwinden. Damit schaffen sie neue Intransparenz, da die Mechanismen, mit denen sie Transparenz ermöglichen, selbst nicht nachvollziehbar sind. Auch das ist eine Kehrseite von Transparenz.

Zum einen werden die Grenzen der Beobachtbarkeit durch das digitale Erfassen menschlicher Aktivitäten verschoben, indem durch automatisierte Prozesse Datenmengen und -inhalte erfasst werden, die menschlicher Beobachtung unzugänglich sind. Zum anderen verschieben sich dadurch die Grenzen der Informationsverarbeitung. Dem wird versucht, durch Informationsfilterung und -verdichtung zu begegnen. Letztlich bekommt der Nutzer nur noch Informationen in konzentrierter grafischer Form, die das System für wichtig erachtet. Eine Auseinandersetzung mit den eigentlichen Beobachtungen kann so nicht mehr stattfinden. Konsequent weitergedacht könnten digitale Ansätze in Zukunft die Grenzen der Handlungsfähigkeit überwinden. Durch detaillierte digitale Hilfen oder mittels autonom handelnder Systeme könnte dem Mitarbeiter als Flaschenhals der Aktivitätskette von der Informationserfassung bis zur anschließenden Handlung eine zunehmend passiv-kontrollierende Rolle zugewiesen werden.

\section{„Digitale Technologien versuchen vollständige Transparenz zu ermöglichen."}

Schon jetzt sind die hinter dieser Automatisierung stehenden Mechanismen der Datenerfassung, die Kriterien für Informationsfilterung und -konzentration sowie die Algorithmen für Vorschlagswesen und autonome Entscheidungen den meisten Personen innerhalb der Organisation weitgehend unzugänglich und unverständlich. Im Extremfall sind sie sogar der Organisation im Gesamten intransparent, da alle Teilaspekte an die Anwendungsanbieter ausgelagert und die Ergebnisse als Service eingekauft werden. Mit dieser zusätzlichen Intransparenz umzugehen ist eine komplett neue Art von Herausforderung für Unternehmen (vergleiche Hosanagar/Jair 2018).

\section{Transparenz ausbalancieren}

Die Dualität von Licht und Schatten digitaler Transparenz erfordert von den Unternehmen Bemühungen um Balance: Balance der Transparenz für ver- 
schiedene Akteure innerhalb der Organisation, Balance zwischen Transparenz und Privatsphäre, Balance zwischen Adaption aller technischer Möglichkeiten und Abwägung der nichttechnischen Konsequenzen. Entscheidend dafür ist die Auseinandersetzung mit diesem Thema bei der Ausgestaltung des Steuerungs- und Kontrollsystems des Unternehmens. Auf die Aspekte Kultur, Leistungsbeurteilung und -entwicklung und technische Umsetzung gehen wir im Folgenden genauer ein.

\section{„Die Dualität von Licht und Schatten digitaler Transparenz erfordert von den Unternehmen Bemühungen um Balance."}

\section{Kultur}

Transparenz ist als Leitwert in der Kultur vieler Unternehmen verankert. Allzu oft ist jedoch nicht klar, was das konkret bedeutet beziehungsweise ob diese Verankerung nur als Grundlage für Forderungen nach Schaffung ebendieser dient. Um dies zu verhindern, sollte Transparenz nicht losgelöst für sich stehen, sondern explizit definiert, begründet und begrenzt werden. Nur wenn die positiven Aspekte der Transparenz explizit von den negativen Aspekten abgegrenzt werden, wird aus dem abstrakten Wert Transparenz ein tatsächlicher Fixpunkt für das Handeln innerhalb der Organisation.

\section{Leistungsbeurteilung und -entwicklung}

Dementsprechend sollte sich der Dreiklang aus Definition, Begründung und Begrenzung auch in der Ausgestaltung der Leistungsbeurteilung und -entwicklung wiederfinden. Im ersten Schritt bedeutet dies eine klare Kommunikation, welche Informationen mit welchem Verwendungszweck transparent gemacht werden und wie Mitarbeiter diese für sich nutzen können. Im zweiten Schritt bedeutet es, in den Prozessen bewusst Privatsphäre für Mitarbeiter zu schaffen. Dies erfordert entweder, ganz auf die Sammlung bestimmter Daten zu verzichten, oder Dritte gezielt von einem Informationszugriff auszuschließen. Nur so kann Transparenz auch eine motivierende Gesamtwirkung entfalten.

\section{Technische Umsetzung}

Im letzten Schritt müssen die so abgegrenzte Transparenz und Privatsphäre fest in der technischen Umsetzung verankert werden. Neben der selektiven Erfassung von Informationen und einer intelligenten User-Trennung bedeutet dies, ein grundsätzliches Verständnis über die Funktionsweise der verschiedenen Systeme bei allen Mitarbeitern zu schaffen und ein tiefgehendes Verständnis innerhalb der Organisation zu bewahren. Um den Bedürfnissen der Mitarbeiter und der Organisation Rechnung zu tragen, sind möglicherweise die Nutzer dabei sogar selbst in die Ausgestaltung ihrer Transparenz- und Privatsphäre-Umgebung eingebunden. Aus einer HR-Perspektive wird so für
Ausgewogen ausgestaltete Steuerungs- und Kontrollsysteme ermöglichen eine bessere Umsetzung von Transparenz.

\section{Handlungsempfehlungen}

- Machen Sie deutlich, was Sie unter Transparenz verstehen, egal ob als Leitwert in Ihrem Unternehmen oder im Rahmen einzelner Maßnahmen.

- Begründen Sie, weshalb und wie Sie die Transparenz erhöhen wollen, gerade wenn die Umsetzung auf digitalen Technologien basiert.

- Setzen Sie der Transparenz klare Grenzen und schaffen Sie somit Raum für die Privatsphäre Ihrer Mitarbeiter. 


\section{Ergänzende Studientipps}

- Bernstein, E. S. (2014): The Transparency Trap, in: Harvard Business Review, 92 (10), S. 1-14, https://tinyurl.com/thetransparency-trap (letzter Abruf: 28.09.2020).

- Birkinshaw, J./Cable, D. (2017): The Dark Side of Transparency, in: McKinsey Quarterly, February, 1-7, https://tinyurl.com/dark-sidetransparency (letzter Abruf: 28.09.2020).

- De Cremer, D. (2016): When Transparency Backfires, and How to Prevent It, in: Harvard Business Review, 21.07.2016, https:// tinyurl.com/how-to-prevent-backfires (letzter Abruf: 17.03.2020).

- Remmers, T./Schäffer, U./Schaupp, D. (2020): Disentangling the Bright and Dark Sides of Transparency - An Integrated Analysis of Psychological Consequences, Working Paper, https://www.ssrn. com/abstract=3708074 (letzter Abruf: 20.10.2020).

- Shah, S./Horne, A./Capellá, J. (2012): Good Data Won’t Guarantee Good Decisions, in: Harvard Business Review, 90 (4), S. 23-25, https://tinyurl.com/data-and-decisions (letzter Abruf: 28.09.2020).

Mitarbeiter ein produktiver Rahmen zur Verbesserung der individuellen Leistungsfähigkeit geschaffen und ein potenziell kontraproduktives TransparenzManagement begrenzt.

\section{Ausblick}

Die Fähigkeit von Organisationen, digitale Transparenz auszubalancieren, gewinnt zunehmend an Bedeutung. Insbesondere der HR-Bereich ist mit neuen Fragen konfrontiert, beispielsweise in Bezug auf die Herausforderungen innerbetrieblicher Transparenz im Bereich Homeoffice. Beschleunigt durch Maßnahmen zur Eindämmung von COVID-19, wird über eine mögliche Verstetigung von Homeoffice-Tätigkeit diskutiert. Dies führt zwangsläufig zu der Frage, wie, wenn überhaupt gewollt, ein Übergreifen in diese privaten Bereiche balanciert werden kann?

\section{Literatur}

Bennis, W. G./ Goleman, D./O’Toole, J. (2008): Transparency. How Leaders Create a Culture of Candor, San Francisco.

Bernstein, E. S. (2017): Making Transparency Transparent. The Evolution of Observation in Management Theory, in: Academy of Management Annals, 11 (1), S. 217-266.

Bernstein, E. S. (2012): The Transparency Paradox. A Role for Privacy in Organizational Learning and Operational Control, in: Administrative Science Quarterly, 57 (2), S. 181-216.

Bhave, D. P./Teo, L. H./Dalal, R. S. (2019): Privacy at Work. A Review and a Research Agenda for a Contested Terrain, in: Journal of Management, 46 (1), S. 127-164.

Deloitte (2016): CFO Survey Frühjahr 2016 - Die neue Realität: digital und volatil, https://tinyurl.com/cfo-survey-1-2016 (letzter Abruf: 05.05.2020). 
Eggers, D. (2015): The Circle, New York.

EIU - The Economist Intelligence Unit (Hrsg.) (2018): The Strategic CFO in a Rapidly Changing World. Survey, https://tinyurl.com/the-strategic-cfo (letzter Abruf: 05.05.2020).

Heald, D. (2006): Varieties of Transparency, in: Hood, C./Heald, D. (Hrsg.): Transparency: the Key to Better Governance? Series: Proceedings of the British Academy, 135, Oxford, S. 25-43.

Hosanagar, K./Jair, V. (2018): We Need Transparency in Algorithms, But Too Much Can Backfire, in: Harvard Business Review, 25.07.2018, https://tinyurl.com/ transparency-backfire (letzter Abruf: 17.03.2020).

Schnackenberg, A. K./Tomlinson, E. C. (2014): Organizational Transparency: A New Perspective on Managing Trust in Organization-Stakeholder Relationships, in: Journal of Management, 42 (7), S. 1784-1810.

\section{SpringerProfessional}

Organizational Transparency

Hofmann, Y. E./Strobel. M. (2020): Transparency Goes a Long Way: Information Transparency and its Effect on Job Satisfaction and Turnover Intentions of The Professoriate, in: Journal of Business Economics, 90, S. 713-732. www.springerprofessional.de/link/17928012

August, V./Osrecki, F. (Hrsg.) (2019): Der Transparenz-Imperativ. Normen - Praktiken Strukturen, Wiesbaden.

www.springerprofessional.de/link/17492146

\section{ICV Controlling Excellence Award 2021}

Du betreust als Controller ein spannendes Projekt und hast damit in deinem Unternehmen neue Akzente gesetzt? Dann reiche dein Projekt für den ICV Controlling Excellence Award 2021 ein.

\section{Durchstarter gesucht.}

\section{Zeig, was}

du drauf hast!

\section{(1)}

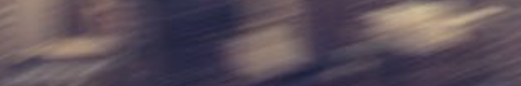

Infos zur Ausschreibung unter www.icv-controlling.com. Einsendeschluss ist der 31. Januar 2021. Verleihung des ICV Controlling Excellence Awards beim 45. Congress der Controller am 26./27. April 2021 in München.

\section{ATVISIO}

C O N S U L T
Sponsoren: C. CA controller

\section{IGC}

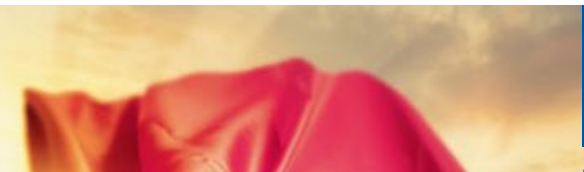
Rer

International

Association of Controllers

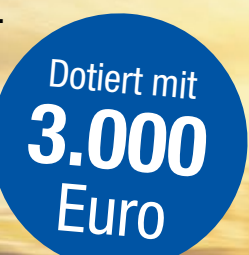

